\title{
Transcriptome profiling of immune response to Yersinia ruckeri in spleen of rainbow trout (Oncorhynchus mykiss)
}

\author{
Di Wang ${ }^{1,2,3}$, Simeng Sun ${ }^{1}$, Shaowu Li $i^{2,3}$, Tongyan $\mathrm{Lu}^{2,3}$ and Dongfang Shi*
}

\begin{abstract}
Background: Yersinia ruckeri is a pathogen that can cause enteric redmouth disease in salmonid species, damaging global production of economically important fish including rainbow trout (Oncorhynchus mykiss). Herein, we conducted the transcriptomic profiling of spleen samples from rainbow trout at $24 \mathrm{~h}$ post-Y. ruckeri infection via RNA-seq in an effort to more fully understand their immunological responses.

Results: We identified 2498 differentially expressed genes (DEGs), of which 2083 and 415 were up- and downregulated, respectively. We then conducted a more in-depth assessment of 78 DEGs associated with the immune system including CCR9, CXCL11, IL-1 $\beta$, CARD9, IFN, TNF, CASP8, NF-KB, NOD1, TLR8a2, HSP90, and MAPK11, revealing these genes to be associated with 20 different immunological KEGG pathways including the Cytokine-cytokine receptor interaction, Toll-like receptor signaling, RIG-l-like receptor signaling, NOD-like receptor signaling, and MAPK signaling pathways. Additionally, the differential expression of 8 of these DEGs was validated by a qRT-PCR approach and their immunological importance was then discussed.
\end{abstract}

Conclusions: Our findings provide preliminary insight on molecular mechanism underlying the immune responses of rainbow trout following $Y$. ruckeri infection and the base for future studies of host-pathogen interactions in rainbow trout.

Keywords: Rainbow trout, Yersinia ruckeri, Spleen, Transcriptome, Immune response

\section{Background}

Yersinia ruckeri is a pathogen that can cause enteric redmouth disease (ERM) or yersiniosis, resulting in significant mortality and economic losses associated with the global production of rainbow trout (Oncorhynchus mykiss). Rainbow trout are highly susceptible to ERM, although other species of fish can also be affected by this disease $[1,2]$. Multiple studies have sought to clarify the immunological responses of fish species to $Y$. ruckeri infection [3, 4]. In one study, Raida et al. determined that very susceptible trout species exhibited a robust and rapid-onset septicemic response to infection associated

\footnotetext{
*Correspondence: shidf@neau.edu.cn

${ }^{1}$ College of Veterinary Medicine, Northeast Agricultural University, 150030 Harbin, China

Full list of author information is available at the end of the article
}

with the production of high levels of pro-inflammatory cytokines [5]. Similarly, these pro-inflammatory cytokines were also upregulated in the spleen of the vaccinated rainbow trout following $Y$. ruckeri challenge, albeit to a lesser extent than in naive fish [6]. The spleen is a key secondary lymphoid organ that is thus closely associated with rainbow trout responses to $Y$. ruckeri infection, and significant changes in the expression of splenic immune-related genes have been detected following $Y$. ruckeri challenge [7, 8]. However, no systematic analyses of patterns of rainbow trout splenic gene expression after $Y$. ruckeri infection have been conducted to date.

RNA sequencing (RNA-seq) is a high-throughput approach to analyzing transcriptomes that has frequently been employed in studies of fish species [9]. Several recent studies based on RNA-Seq analysis have explored 
rainbow trout responses to a range of pathogen types, such as splenic responses to Aeromonas salmonicida $[10,11]$, infectious hematopoietic necrosis virus (IHNV) [12], and Ichthyophthirius multifiliis [13]. Such transcriptomic analyses have offered new insights into the etiology of these diseases, and similar studies of $Y$. ruckeri infections may highlight viable approaches for treating or preventing yersiniosis in rainbow trout farming.

As such, we herein conducted a transcriptomic study assessing rainbow trout splenic immune responses to $Y$. ruckeri infection. After identifying infection-related differentially expressed genes (DEGs), we validated a subset of these genes via GRT-PCR and conducted the functional annotation of immune-associated DEGs. Together, our data offer a preliminary insight for future research regarding the immunological mechanisms involved in rainbow trout defensive response against $Y$. ruckeri.

\section{Results}

\section{RNA-sequencing and data processing}

Genes associated with rainbow trout immune response to $Y$. ruckeri infection were identified by assessing spleen samples from YR-infected and control uninfected fish via RNA-sEq. In total, six cDNA libraries were prepared (from 3 per group), and raw data were generated (Table S1) and deposited in the NCBI Sequence Read Archive (SRA) under accession number SRR13014589 SRR13014594.

Following the completion of filtering, $44.07 \mathrm{G}$ bp of clean data were extracted, with over $93.15-93.55 \%$ of the bases reads having a phred quality value $\geq 30$ in the non-infected group compared to $92.87-93.43 \%$ in the YR-infected group. These quality scores were consistent with excellent quality data. Reads from these two groups exhibited GC contents of $49.14-49.64 \%$ and $49.00-$ $49.18 \%$, respectively (Table 1 ).

The total number of expressed genes detected in samples from uninfected rainbow trout was slightly higher than that detected in YR-infected rainbow trout (Fig. 1).

\section{Read mapping to the reference genome}

Cleaned reads were mapped to the $O$. mykiss reference genome, with $84.81-85.99 \%$ of these reads ultimately matching perfectly. Over $70 \%$ of reads aligned to exonic regions in each library, of which $78.05-78.24 \%$ in the uninfected group and $78.53-79.11 \%$ in the YR-infected groups mapping to unique reads whereas $6.76-7.38 \%$ in the uninfected group and $6.81-7.17 \%$ in the YR-infected groups mapping to multiple reads. A total of 123.7985 $(41.90 \%)$ and $125.0329(42.32 \%) \mathrm{M}$ reads in the uninfected and YR-infected groups mapped to reference genome sense and antisense strands, respectively (Table 2 ). Besides, some new genes were detected and classified with the NR, Swiss-Prot, GO, COG, KOG, Pfam, and KEGG databases (Table S2).

\section{DEG identification and analysis}

The Pearson's correlation coefficient values were used to assess relative gene expression in the uninfected and YR-infected groups (Fig. S1). A total of 2498 DEGs were identified by comparing these groups, of which 2083 $(83.39 \%)$ were up-regulated and 415 (16.61\%) were down-regulated, in YR-infected fish compared to uninfected fish (Table S3). Volcano and MA plots were also used to represent these gene expression trends (Fig. S2).

Of these DEGs, 2431 were classified successfully using the NR, Swiss-Prot, GO, COG, KOG, Pfam, and KEGG databases (Table 3). With respect to new genes, many DEGs were annotated using the NR and eggNOG databases, but few were annotated in the COG database.

To better understand the functional roles of detected DEGs, GO annotation was next performed by categorizing these DEGs into 23 biological processes (BPs), 19 cellular components (CCs), and 16 molecular functions (MFs). Cellular (42.07\%), single-organism (36.51\%), metabolic $(30.75 \%)$, and biological $(29.64 \%)$ processes were the most dominant categories of BPs, while

Table 1 Characteristics of RNA-seq data

\begin{tabular}{|c|c|c|c|c|}
\hline Samples & Clean reads $(\mathrm{M})$ & Clean bases (Gb) & GC Content (\%) & Q30 (\%) \\
\hline non-infected rainbow trout 1 & 26.9191 & 8.0181 & 49.14 & 93.18 \\
\hline non-infected rainbow trout 2 & 23.4555 & 6.9991 & 49.64 & 93.55 \\
\hline non-infected rainbow trout 3 & 25.5238 & 7.6123 & 49.33 & 93.15 \\
\hline YR-infected rainbow trout 1 & 22.5961 & 6.7523 & 49.18 & 92.87 \\
\hline YR-infected rainbow trout 2 & 23.5341 & 7.0248 & 49.00 & 93.43 \\
\hline YR-infected rainbow trout 3 & 25.7487 & 7.6694 & 49.18 & 93.32 \\
\hline Non-infected group & 75.8984 & 22.6295 & 49.32 & 93.29 \\
\hline YR-infected group & 72.0286 & 21.4465 & 49.12 & 93.21 \\
\hline Total & 147.927 & 44.076 & 49.25 & 93.25 \\
\hline
\end{tabular}




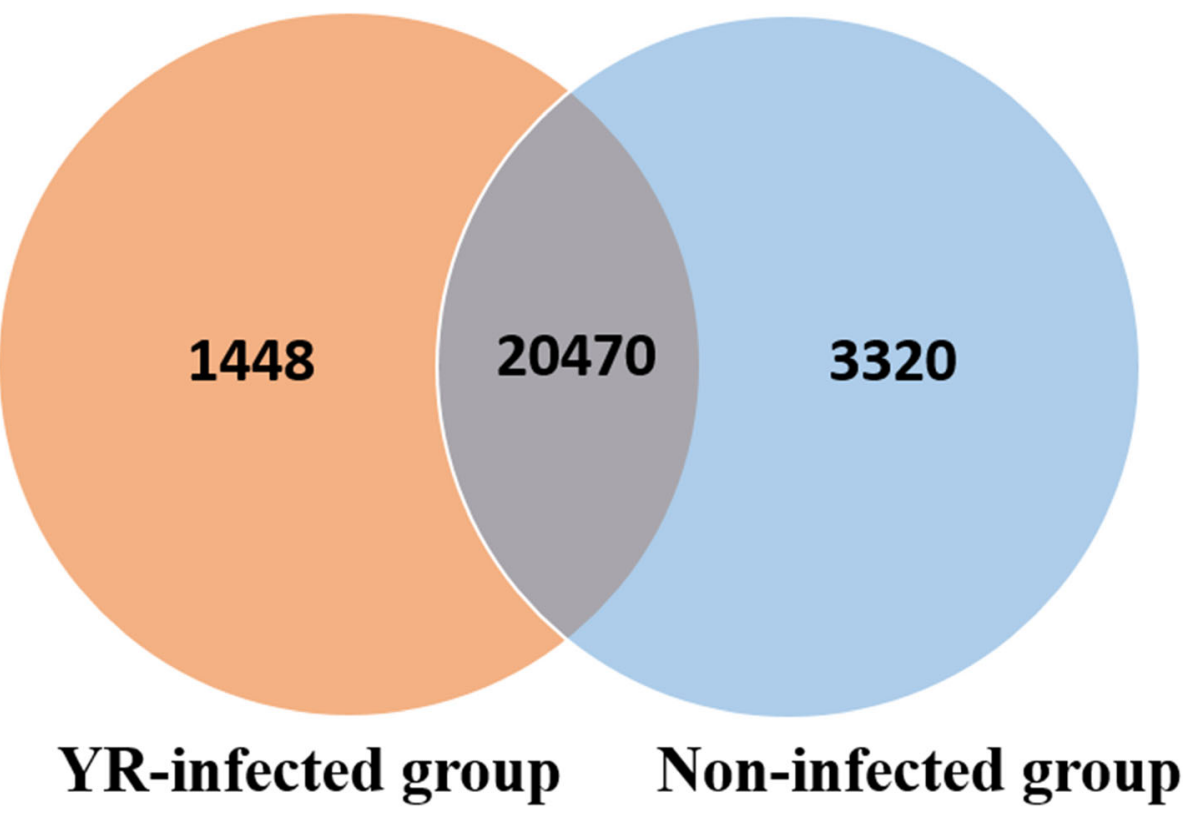

Fig. 1 A Venn diagram indicating the numbers of genes detected in YR-infected and uninfected rainbow trout spleen samples

membrane $(27.94 \%)$, cell $(26.34 \%)$, cell part $(25.66 \%)$, and membrane part $(24.70 \%)$ were the most enriched CCs and binding (40.07\%) and catalytic activity (19.26\%) were the most dominant MFs (Fig. 2).

In addition, KEGG pathway enrichment analyses were performed to assess the functional roles of these DEGs during $Y$. ruckeri infection in rainbow trout. Assembled DEGs were analyzed with the KEGG database, leading to their classification into 6 categories (Fig. S3). KEGG enrichment results, including the top 9 pathways enriched for $>50$ genes $(P<0.05)$, are shown in Fig. 3. Four highly enriched pathways were detected through this KEGG analysis, including the NOD-like receptor signaling, cytokinecytokine receptor interaction, Toll-like receptor signaling, and RIG-I-like receptor signaling pathways. The preferential enrichment of these pathways suggests that many of the genes differentially expressed between uninfected and YRinfected rainbow trout were related to the immune system.

Table 2 RNA-seq alignment details and mapping ratios

\begin{tabular}{|c|c|c|c|c|c|c|}
\hline Samples & Total reads $(\mathrm{M})$ & $\begin{array}{l}\text { Mapped } \\
\text { reads (M) }\end{array}$ & $\begin{array}{l}\text { Uniq mapped } \\
\text { reads }(M)\end{array}$ & $\begin{array}{l}\text { Multiple map } \\
\text { reads (M) }\end{array}$ & Reads map to ' + ' & Reads map to ' \\
\hline Non-infected rainbow trout 1 & 53.8382 & $45.6616(84.81 \%)$ & $42.0247(78.06 \%)$ & $\begin{array}{l}3.6369 \\
(6.76 \%)\end{array}$ & $22.4170(41.64 \%)$ & $22.6348(42.04 \%)$ \\
\hline Non-infected rainbow trout 2 & 46.9109 & 40.1607 (85.61 \%) & $36.7008(78.24 \%)$ & $\begin{array}{l}3.4599 \\
(7.38 \%)\end{array}$ & $19.7468(42.09 \%)$ & $19.9220(42.47 \%)$ \\
\hline Non-infected rainbow trout 3 & 51.0477 & $43.3188(84.86 \%)$ & $39.8427(78.05 \%)$ & $\begin{array}{l}3.4761 \\
(6.81 \%)\end{array}$ & $21.3261(41.78 \%)$ & $21.4872(42.09 \%)$ \\
\hline YR-infected rainbow trout 1 & 45.1922 & $38.7941(85.84 \%)$ & $35.5522(78.67 \%)$ & $\begin{array}{l}3.2418 \\
(7.17 \%)\end{array}$ & 19.0209 (42.09\%) & $19.2198(42.53 \%)$ \\
\hline YR-infected rainbow trout 2 & 47.0682 & $40.4739(85.99 \%)$ & $37.2354(79.11 \%)$ & $\begin{array}{l}3.2386 \\
(6.88 \%)\end{array}$ & 19.7989 (42.06 \%) & $20.0358(42.57 \%)$ \\
\hline YR-infected rainbow trout 3 & 51.4973 & $43.9318(85.31 \%)$ & $40.4383(78.53 \%)$ & $\begin{array}{l}3.4934 \\
(6.78 \%)\end{array}$ & $21.4897(41.73 \%)$ & $21.7333(42.20 \%)$ \\
\hline Non-infected group & 50.5989 & $\begin{array}{l}129.1411 \\
(85.09 \%)\end{array}$ & $\begin{array}{l}118.5682 \\
(78.12 \%)\end{array}$ & $\begin{array}{l}10.5729 \\
(6.98 \%)\end{array}$ & $\begin{array}{l}63.4899 \\
(41.84 \%)\end{array}$ & $\begin{array}{l}64.0440 \\
(42.20 \%)\end{array}$ \\
\hline YR-infected group & 47.9192 & $\begin{array}{l}123.1998 \\
(85.71 \%)\end{array}$ & $\begin{array}{l}113.2259 \\
(78.77 \%)\end{array}$ & $\begin{array}{l}9.9738 \\
(6.94 \%)\end{array}$ & $\begin{array}{l}60.3095 \\
(41.96 \%)\end{array}$ & $\begin{array}{l}60.9889 \\
(42.43 \%)\end{array}$ \\
\hline Total & 295.5624 & $\begin{array}{l}252.3409 \\
(85.40 \%)\end{array}$ & $\begin{array}{l}231.7941 \\
(78.44 \%)\end{array}$ & $\begin{array}{l}20.5467 \\
(6.96 \%)\end{array}$ & $\begin{array}{l}123.7985 \\
(41.90 \%)\end{array}$ & $\begin{array}{l}125.0329 \\
(42.32 \%)\end{array}$ \\
\hline
\end{tabular}


Table 3 Summary statistics regarding DEG functional annotation

\begin{tabular}{|c|c|c|c|c|c|c|c|c|c|}
\hline Annotated databases & NR & Swiss-Prot & GO & COG & KOG & Pfam & KEGG & eggNOG & All \\
\hline DEGs number & 2421 & 1679 & 1766 & 584 & 1539 & 2116 & 1533 & 2295 & 2431 \\
\hline Ratio (\%) & 99.59 & 69.07 & 72.65 & 24.02 & 63.31 & 87.04 & 63.06 & 94.41 & \\
\hline
\end{tabular}

\section{Identification of immune-related DEGs}

To better understand the intracellular signaling pathways during $Y$. ruckeri infection in rainbow trout, we therefore focused on 78 immune response-related DEGs identified in this study, including two new genes (Table S4). A heatmap was constructed based upon the fold-change expression values for these DEGs (Fig. 4), clearly demonstrating that almost all of these genes (74) were upregulated in spleen samples from YR-infected fish compared to spleen samples from uninfected fish, whereas only 4 genes were down-regulated after infection.

Further analysis of these immune-related DEGs revealed them to be primarily associated with 20 immunological KEGG pathways, including the MAPK signaling, Cytokine-cytokine receptor interaction, Toll-like receptor signaling, RIG-I-like receptor signaling, NOD-like receptor signaling, FoxO signaling, mTOR signaling, apoptosis, TGF-beta signaling, regulation of autophagy, ErbB signaling, cell adhesion molecule (CAM), intestinal immune network for IgA production, cytosolic DNAsensing, phosphatidylinositol signaling system, and p53 signaling pathways (Table 4). The top 3 pathways enriched in these genes included the NOD-like receptor signaling (31 genes), RIG-I-like signaling (35 genes), and Toll-like receptor signaling (51 genes) pathways (Fig. 5).

\section{Validation of selected DEGs by qRT-PCR}

As expected, all the eight immune-related DEGs exhibited similar expression trends when measured via both qPCR and RNA-Seq analysis, confirming the reliability of our analytical techniques (Fig. 6).

\section{Discussion}

ERM is a serious disease that impacts global salmonid populations [14]. While some studies have begun to characterize rainbow trout immune responses to $Y$. ruckeri infection [8, 15], no systematic transcriptomic analyses of these responses have been conducted to date. The spleen plays central roles in orchestrating innate and adaptive immune responses in fish. Herein, we sequenced the spleen transcriptomes of rainbow trout infected with YR in comparison with those of control uninfected rainbow trout and we identified 2498 DEGs between these populations, of which 2083 were upregulated whereas 415 were down-regulated in infected rainbow trout. Immune response-related DEGs were then assessed in additional detail in an effort to explore the basis of immune responses against $Y$. ruckeri infection in rainbow trout.

Cytokines are secreted by a range of cell types, and they act as immune response regulators that can be

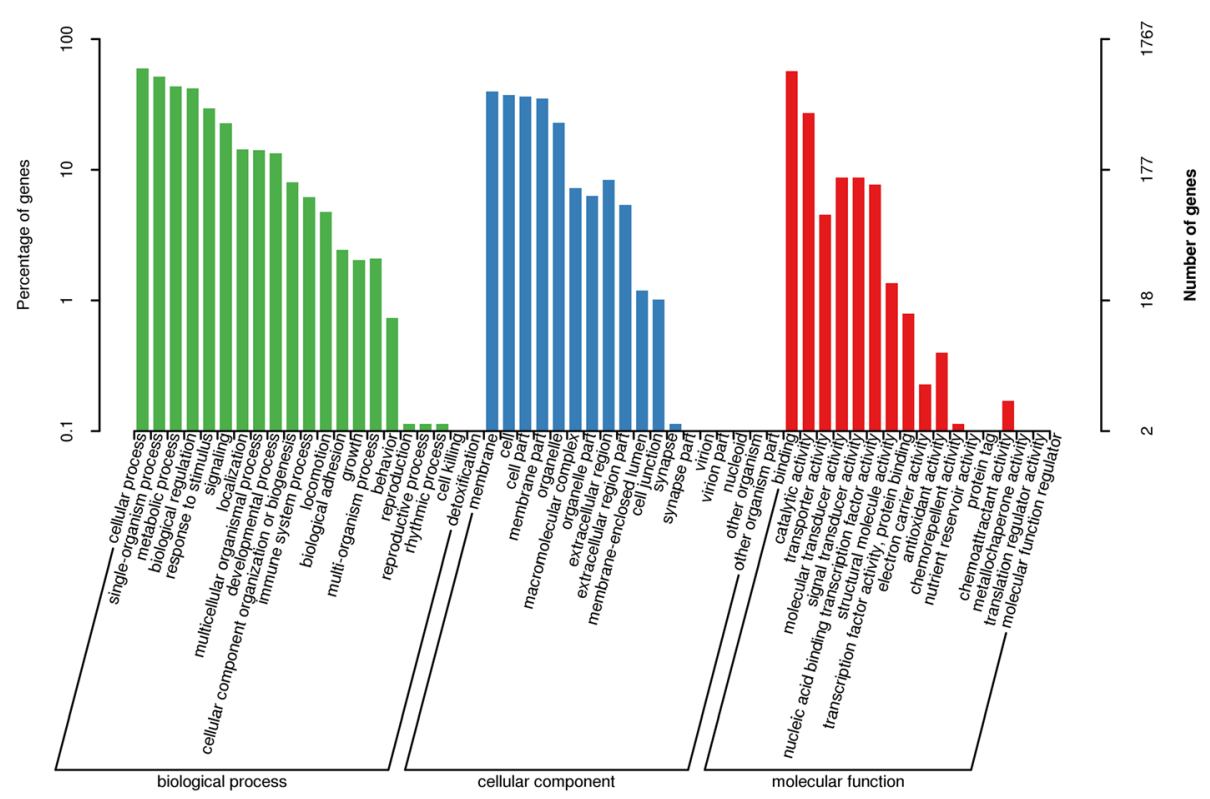

Fig. 2 GO annotation of DEGs. DEGs were classified based on their enrichment in specific biological processes, cellular components, and molecular functions 

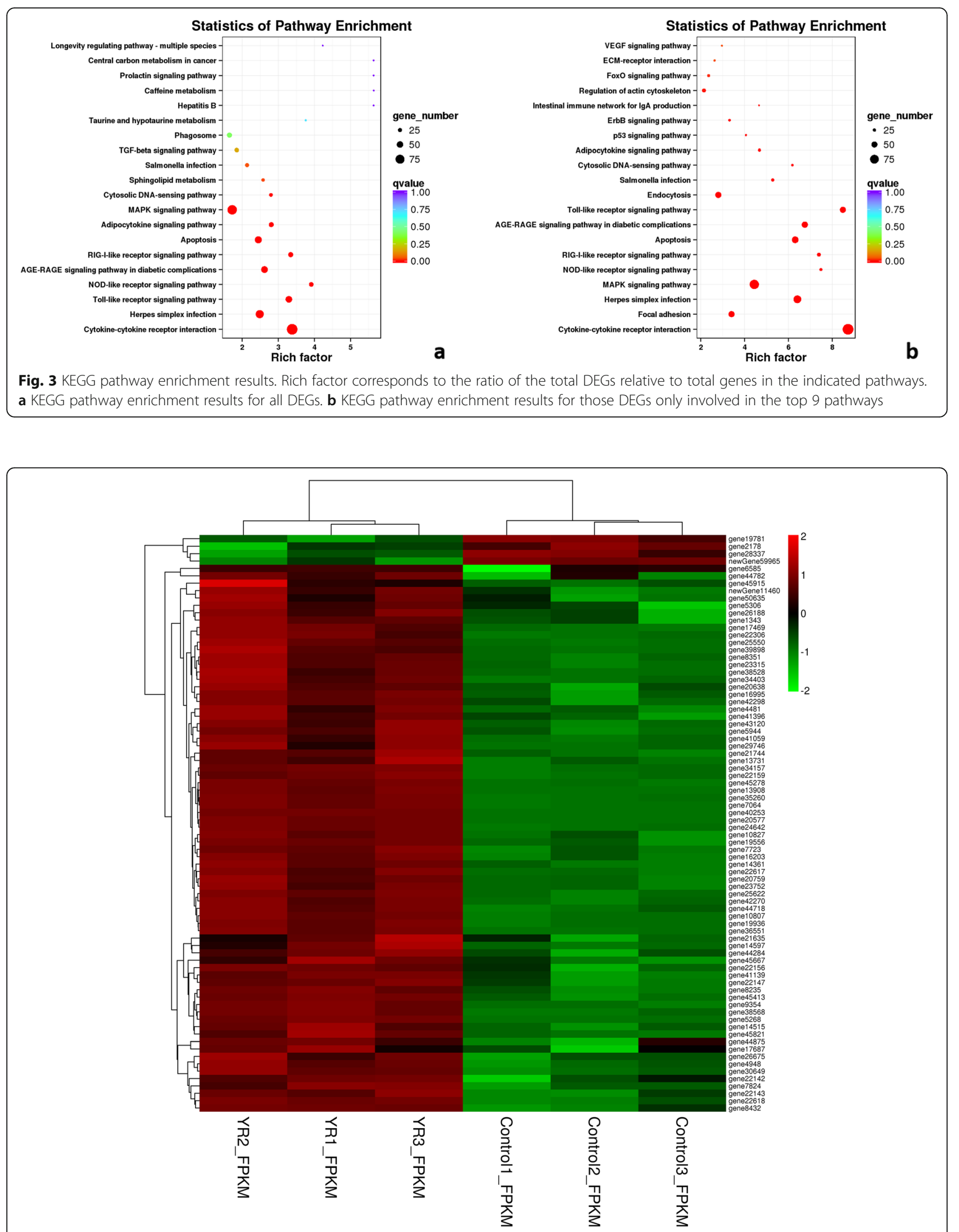

Fig. 4 Immune-related DEGs in the non-infected and YR-infected rainbow trout 
Table 4 Immune-related DEGs

\begin{tabular}{llcll}
\hline Gene ID & Type & Log $_{2}$ Fold & Putative homolog protein & KEGG pathway \\
\hline Gene 10,807 & up & 12.0338 & Interleukin-1 beta & ko04620: Toll-like receptor signaling pathway \\
Gene 24,642 & up & 10.6682 & Interleukin-8 & ko04060: Cytokine-cytokine receptor interaction \\
Gene 22,618 & up & 9.3812 & Interleukin-8 & ko04621: NOD-like receptor signaling pathway \\
Gene 4948 & up & 8.4892 & Tumor necrosis factor & ko04150: mTOR signaling pathway \\
Gene 28,337 & down & -2.5818 & Mitogen-activated protein kinase 11 & ko04010: MAPK signaling pathway \\
Gene 25,622 & up & 10.1562 & Interleukin-6 & ko04060: Cytokine-cytokine receptor interaction \\
Gene 34,157 & up & 9.0084 & Interleukin-6 & ko04060: Cytokine-cytokine receptor interaction \\
Gene 34,403 & up & 6.5996 & Tumor necrosis factor & k004060: Cytokine-cytokine receptor interaction \\
Gene 25,550 & up & 6.1699 & Tumor necrosis factor & ko04060: Cytokine-cytokine receptor interaction \\
Gene 22,142 & up & 3.1333 & Interferon & k004060: Cytokine-cytokine receptor interaction \\
Gene 2178 & down & -1.1786 & NOD1 & ko04621: NOD-like receptor signaling pathway \\
Gene 20,638 & up & 4.6720 & Small cytokines (intecrine/chemokine) & ko04060: Cytokine-cytokine receptor interaction \\
newGene59965 & down & -1.4640 & Toll-like receptor 8 & ko04620: Toll-like receptor signaling pathway \\
Gene 26,188 & up & 3.7094 & Mab-21 protein & ko04623: Cytosolic DNA-sensing pathway \\
Gene 44,284 & up & 3.1644 & Immunoglobulin V-set domain & ko04514: Cell adhesion molecules (CAMs) \\
Gene 23,752 & up & 2.9294 & Phosphoinositide 3-kinase regulatory subunit & ko04012: ErbB signaling pathway \\
Gene 5944 & up & 2.4306 & Interferon alpha/beta receptor & ko04060: Cytokine-cytokine receptor interaction
\end{tabular}

classified as interleukins (ILs), interferons (IFNs), tumor necrosis factors (TNFs), and chemokines [16]. Of the 78 immune-associated DEGs in the present study, 31 were classified into the cytokine-cytokine receptor interaction pathway, including chemokine ( $\mathrm{C}-\mathrm{X}-\mathrm{C}$ motif) ligand
(CXCL11), C-C motif chemokine receptor 9 (CCR9), caspase recruitment domain-containing protein (CARD9), $I L-12, I L-1 \beta, I F N$ and TNF. Chemokines control the migration of particular immune cell subsets and coordinate both adaptive and innate immune responses to stressors

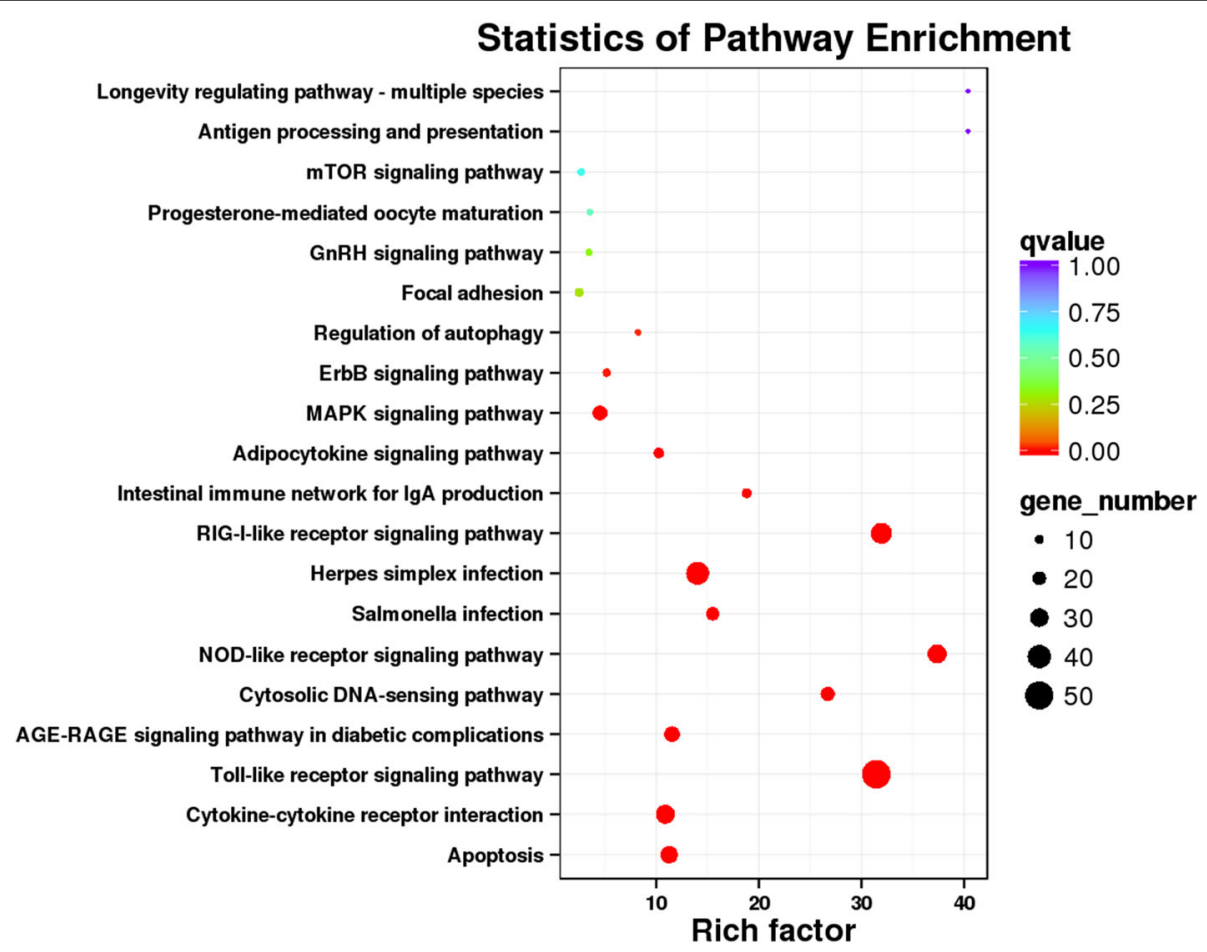

Fig. 5 KEGG pathways enriched in genes differentially expressed between uninfected and YR-infected rainbow trout 


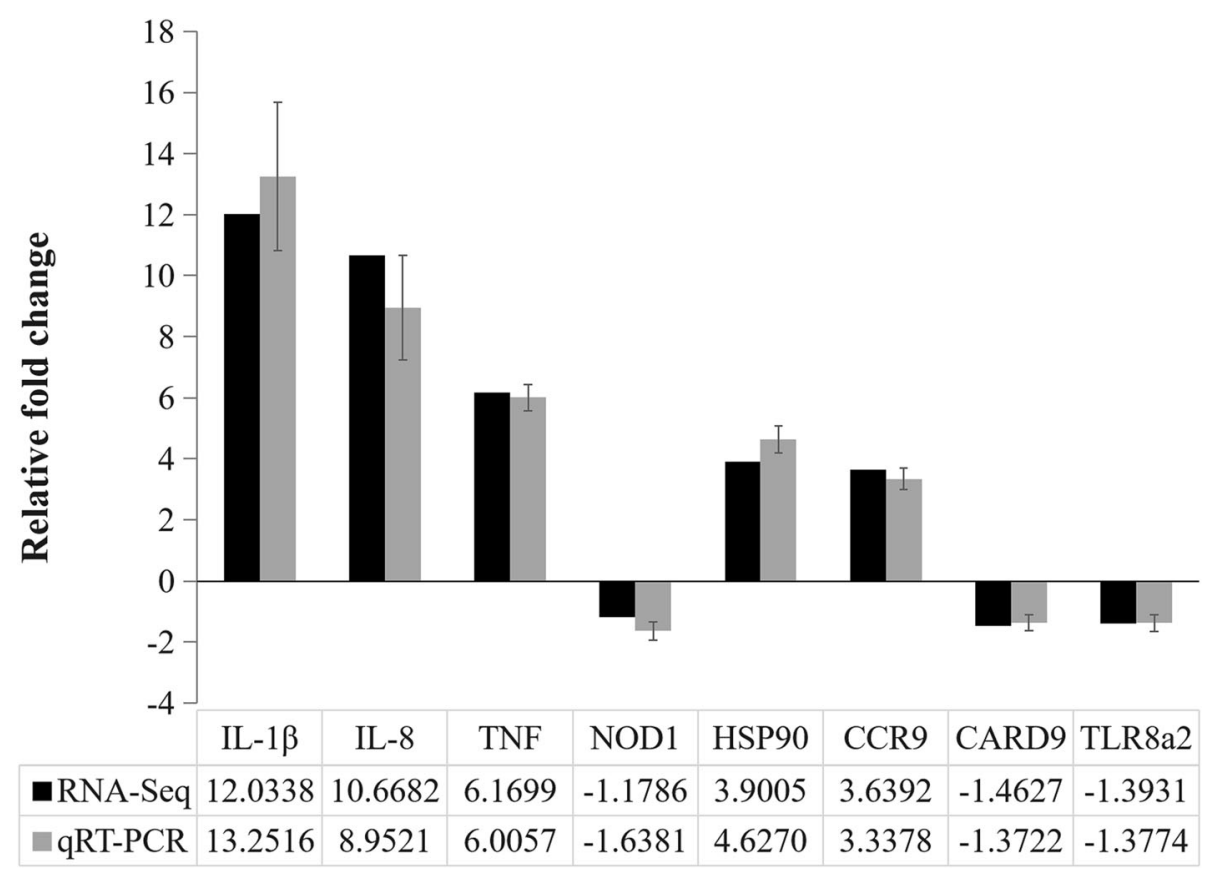

Fig. 6 Comparison of DEG expression in GPCR and RNA-seq analyses. Relative gene expression levels were normalized to EF-1a

[17]. The transcription of $C X C d$ in rainbow trout has previously been shown to be induced in response to $Y$. ruckeri infection [18]. Herein, we observed the upregulation of both CXCL11 and CCR9 in the spleens of rainbow trout infected with this bacterium, consistent with the pathogen-induced chemokine regulation. CARD9, which is normally activated by CLRs [19], was 1.39 -fold downregulated in response to $Y$. ruckeri. Zuo et al. [8] investigated the immune gene expression in rainbow trout to $Y$. ruckeri infection by qRT-PCR and indicated that the genes encoding inflammatory cytokines $(I L-1 \beta$, $I L-2 \quad A, I L-6$ A, IL-8, IL-10 A,IL-12, IL-17 A/F2A, IL$17 C 1, I L-17 C 2, I L-22, T N F \alpha$ ) were generally upregulated in spleen, gills and liver. Our findings also showed the same results on the cytokines expression during $Y$. ruckeri infection, suggesting involvement of these immunerelated genes in response of rainbow trout to bacterial infection (Table 4).

Apoptosis is an important determinant of cellular survival in both physiological and pathological contexts, and can be triggered by factors such as hypoxia, chemical exposure, temperature stress, or immune responses to particular stimuli. Upon bacterial infection, a host's cells may undergo apoptotic death to mitigate the spread of the pathogen within host tissues [20]. Herein, we observed the upregulation of caspase 8 (CASP8), receptor-interacting serine/threonine-protein kinase 1like (RIPK1) and NF-kappa-B inhibitor alpha-like $(I \kappa B \alpha)$ following YR infection in rainbow trout. Caspases are proteases that serve as essential regulators of apoptotic cell death, with CASP8 having showed to be an upstream regulator of apoptotic cascades in fish [21]. Marked CASP8 upregulation has also previously been detected in head-kidney and spleen leukocytes of Totoaba macdonaldi at 24 h post-infection with Vibrio parahaemolyticus and Aeromonas veronii [22]. RIPK1 was identified as a central driver of inflammation in atherosclerosis by its ability to activate the $N F-\kappa B$ pathway and promote inflammatory cytokine release in mice (Mus musculus) [23]. $N F-\kappa B$ can control innate and adaptive immunerelated gene expression, inducing apoptosis in response to numerous stimuli [24]. At the same time, $N F-\kappa B$ activation induces $I \kappa B \alpha$ expression in rainbow trout, in turn resulting in the feedback inhibition of $N F-\kappa B$ [25]. Upregulation of $I k B \alpha, I A P s$ and RIPK1 detected in this study can suggest the compensatory activation of some inhibitors of apoptotic cell death, underscoring the complexities of cellular responses to $Y$. ruckeri in rainbow trout. Additional work must be done in order to understand in depth how the apoptotic processes.

Pattern recognition receptors (PRRs) serve as innate sensors that can rapidly detect and respond to conserved damage- and pathogen-associated molecular patterns (DAMPs and PAMPs, respectively), resulting in the induction of immune-related gene expression and antipathogen responses. PRRs detected in aquatic species to date include TLRs, NLRs, RLRs, and CLRs [26]. In the present study, we identified several DEGs belonging to TLR, NLR, and RLR gene families in the spleens of rainbow trout at $24 \mathrm{~h}$ post- $Y$. ruckeri infection, including 
nucleotide-binding oligomerization domain-containing protein 1-like (NOD1), toll-like receptor $8 \alpha 2$ (TLR8 $\alpha 2)$, etc. NOD1 modulates the innate immune response of fish to bacterial peptidoglycan. Loss- and gain-offunction experiments have suggested that NOD1 can control rainbow trout pro-inflammatory cytokines in rainbow trout [27]. Palti et al. first reported the presence of the TLR8 2 gene in rainbow trout, which they found to be somewhat downregulated in response to treatment with the human agonist of TLR7/8 known as R848 [28]. Here we found that both NOD1 and TLR8 22 were downregulated in rainbow trout spleen during the early stages of $Y$. ruckeri infection. KEGG pathway analysis indicated that many DEGs were involved in TLR signaling pathway, NLR signaling pathway and RLR signaling pathway, such as heat shock protein 90 (HSP90), tumor necrosis factor alpha-induced protein 3-like (TNFAIP3), transcription factor AP-1, IL-1, IL-12, NF- $K B, R I P K 1$, CASP8 and so on. HSPs are important regulators of fish immune responses [29, 30], and HSP90 upregulation detected in the present research may be linked to the rainbow trout innate immune defenses to $Y$. ruckeri infection. Of interest, a pathogen-specific expression pattern of HSP9O was observed in channel catfish (Ictalurus punctatus) and it showed different expression patterns following Flavobacterium columnare and Edwardsiella ictaluri infection [31]. TNFAIP3 (A20) acts as a negative feedback regulator of RIG-I pathway for the establishment of an antiviral state in teleost. TNFAIP3 interrupted RIG-I signaling at the level of TBK1 kinase, a critical point of convergence for many different pathways that activates important transcription factors involved in the expression of many cytokines [32]. In the present study, TNFAIP3 was found to be upregulated after $Y$. ruckeri infection, demonstrating that this gene was involved in immune response of rainbow trout during bacterial infection. Overall, these findings suggest that the PRRs were differentially expressed in rainbow trout and may be important mediators of the initial induction of immunological responses to bacterial infection.

The MAPK signaling pathway is responsive to diverse extracellular stimuli and can modulate transcription factor expression and activation, controlling a range of biological processes including proliferation, apoptosis, and gene transcription. Recent evidence indicates that fish MAPKs can be induced by a range of stimuli. For example, flagellin treatment is associated with MAPK11 upregulation in the head kidney of rock bream (Oplegnathus fasciatus) [33]. In contrast, in the present study we observed a 2.58-fold decrease of MAPK11 expression in the spleen of rainbow trout following $Y$. ruckeri infection, although additional validation of these results is warranted. MAPK8 (Jnk1) has been identified in many fish species and its expression pattern varies by different stimuli [34, 35]. Infection with Aeromonas hydrophila and Bacillus subtilis could induce significant expressions of the jnk1 gene in Labeo rohita [36]. We also observed a significant MAPK8 upregulation upon Y. ruckeri infection in rainbow trout, suggesting that these MAPKs might play a crucial role during the bacterial pathogenesis in rainbow trout. Moreover, a total of 22 DEGs involved in the MAPK signaling pathway seems to play key roles in the rainbow trout response to infection with this bacterium.

\section{Conclusions}

In summary, we conducted a transcriptomic analysis of spleen samples from rainbow trout infected with $Y$. ruckeri in an effort to better understand the immunological basis for responses to this pathogen, leading to the identification of several key immune-related DEGs. Overall, our results will provide a preliminary insight on the immune responses of rainbow trout following $Y$. ruckeri infection and the base for future studies of hostpathogen interactions in rainbow trout.

\section{Methods}

\section{Experimental fish and bacteria}

Healthy rainbow trout $(\sim 10 \mathrm{~g})$ were obtained from Benxi Agrimarine Industries Inc. and maintained in a $540 \mathrm{~L}$ fiberglass circulating water tank at a constant temperature of $14 \pm 0.2^{\circ} \mathrm{C}$ with a $12 \mathrm{~h}$ light/dark cycle and an $8.0 \mathrm{mg} / \mathrm{L}$ oxygen saturation. Fish were maintained under these conditions for 2 weeks and were fed commercial rainbow trout feed.

Y. ruckeri strain BH1206 was isolated from infected rainbow trout, confirmed to be pathogenic, and used for challenge experiments as previously published [37]. Bacteria were grown for $24 \mathrm{~h}$ in TSB medium (BD Difco, USA) and collected by spinning for $5 \mathrm{~min}$ at $6,000 \mathrm{xg}$ prior to resuspension in sterile PBS $(\mathrm{pH} 7.2)$ at $6 \times 10^{7}$ $\mathrm{CFU} \cdot \mathrm{mL}^{-1}$.

\section{Bacterial challenge and sampling}

Prior to challenge test, healthy rainbow trout were kept under laboratory conditions in flow-through tanks at approximately $14{ }^{\circ} \mathrm{C}$ with continuous aeration and fed twice a day at $1.2 \%$ of body weight with commercial fish feed. A subset of experimental fish was microscopically and bacteriologically examined to verify freedom of $Y$. ruckeri infection. Tricaine methanesulfonate (MS222) was used to anesthetize fish prior to the challenge or tissue sample collection. For the challenge test, the experimental fish were kept in two tanks with 6 fish in each tank under the same conditions for fish acclimation. Experimental infection was induced by intraperitoneally (i.p.) injecting fish with $100 \mu \mathrm{L}$ of BH1206 bacteria at $6 \times 10^{5} \mathrm{CFU}$ per gram of fish body weight. An equivalent 
volume of PBS was injected into uninfected control fish. At $24 \mathrm{~h}$ post-infection, three fish per group were sacrificed by an overdose of anesthetic, and spleens were collected, washed to remove blood and fat, snap-frozen with liquid nitrogen, finally stored in liquid nitrogen tank. To confirm the presence of $Y$. ruckeri in experimental fish, the kidney was sampled to perform bacteriological examination.

\section{RNA isolation}

Splenic RNA was isolated using Trizol (Invitrogen, USA), after which RNA integrity and purity were evaluated via $1 \%$ agarose gel electrophoresis and using an Agilent 2100 Bioanalyzer (Agilent Technologies, CA, USA), while a Qubit RNA Assay Kit and a Qubit 2.0 Fluorometer (Life Technologies, CA, USA) were utilized to measure RNA concentration. After RNA preparation, all downstream library preparation and sequencing were performed by Biomarker technologies CO., LTD (Beijing, China).

\section{Library construction and sequencing}

A total of $3 \mu \mathrm{g}$ RNA per spleen sample was utilized for library construction using a NEBNext Ultra RNA Library Prep kit for Illumina (NEB, USA), with samples being affixed with appropriate barcodes. Following DNase I treatment, the remaining mRNA was purified and sheared into 200-250 bp fragments as discussed previously [38]. Library quality was assessed with an Agilent Bioanalyzer 2100 instrument, and a cBot Cluster Generation System with TruSeq PE Cluster Kit v4-cBot-HS (Illumina) was used to cluster barcoded samples. An Illumina Hiseq 2500 platform was then used for the paired-end sequencing of these prepared library samples.

\section{Data processing}

Raw data were initially cleaned by removing reads that contained adapter sequences, poly- $\mathrm{N}$ sequences, and lowquality reads with the FastQC program (http://www. bioinforatics.babraham.ac.uk/projects/fastqc/), after which clean data Q30, GC-content, and sequence duplication levels were calculated. The Trinity software [39] was then used to assemble reads into EST clusters, followed by de novo assembly and alignment to the rainbow trout reference genome (http://www.genoscope.cns.fr/trout/data/) with TopHat (v.2.0.5). Functional annotation was performed by comparing unigenes to the following databases: $\mathrm{Nr}$ (NCBI non-redundant protein sequences); Nt (NCBI non-redundant nucleotide sequences);Pfam (Protein family); KOG/COG (Clusters of Orthologous Groups of proteins) [40]; Swiss-Prot (A manually annotated and reviewed protein sequence database); KO (KEGG Ortholog database) [41]; GO (Gene Ontology) [42].

\section{DEGs identification}

The RSEM software was used to assess unigene expression based upon reads per kilobase of exon per million mapped reads (RPKM) [43]. The DESeq $R$ package (1.10.1) was used to identify DEGs between infected and non-infected fish using a negative binomial distributionbased model, with $P$ values being adjusted as indicated by the Benjamini and Hochberg approach to reduce the false discovery rate. DEGs were considered as those genes with an adjusted $P$-value $<0.05$, and were represented with volcano and MA plots. The Kyoto Encyclopedia of Genes and Genomes (KEGG) database was used for functional enrichment analysis of DEGs, with pathways that had a Q-value $\leq 0.05$ after correcting for multiple testing being considered significantly enriched [44-46]. Furthermore, the immune-related DEGs were selected by mapping the ' 5.1 Immune system' in KEGG maps (https://www.genome.jp/kegg/ pathway.html).

\section{Validation of immune-related DEGs by qRT-PCR}

To confirm the results of RNA-sequencing, eight immune-related DEGs (IL-1 $\beta, I L-8, T N F, N O D 1, C A R D$, TLR8 2 2, CCR9, and HSP90) were randomly selected for qRT-PCR-based validation using the same RNA samples prepared for RNA-seq using primers designed with the Premier primer 5 software (Table 5). EF-1 $\alpha$ was used as a

Table 5 qRT-PCR Primers Lists

\begin{tabular}{|c|c|c|c|}
\hline Gene_ID & Gene name & Forward primer sequence $\left(5^{\prime}-3^{\prime}\right)$ & Reverse primer sequence $\left(5^{\prime}-3^{\prime}\right)$ \\
\hline Gene10807 & $I L-1 \beta$ & CAACTAAGATGGCCGCAAA & TCGGTACATACTCTAAACCTC \\
\hline Gene24642 & IL-8 & ATTTATAAGCTTGATAGGCTG & GTTGTATATAAGAAACCGACT \\
\hline Gene25550 & TNF & CAGGAGCATCACTACCTTC & TTACTAGAACTITCTGCGGAT \\
\hline Gene2178 & NOD1 & ATACAACTGCTACCCCGACCA & AGGCACATTCACCAGGTCCA \\
\hline Gene14361 & HSP9O & GATCCTTCACCGTCAAAGTCG & TCACTTCCTTGTCACGCTCC \\
\hline Gene45413 & CCR9 & ATCTTGAATTTAAGCGCCTGT & ACATCATCCTCACCAACCGTA \\
\hline Gene19781 & CARD9 & TGACAACACTGACACGGAT & ATGCACATGAAGAGATACAAGC \\
\hline newGene59965 & TLR8a2 & CTCTGCCATTTTGATTGGGA & CCCCTAAGAAATCCACGAGA \\
\hline Housekeeping gene & $E F-1 a$ & GATCCAGAAGGAGGTCACCA & TTACGTTCGACCTTCCATCC \\
\hline
\end{tabular}


normalization control for these analyses. SYBR Green dye (Takara, China) and an ABI PRISM 7500 Fast Real-time PCR instrument were used for qRT-PCR based on provided protocols. All reactions were conducted in triplicate with the following thermocycler settings: $60 \mathrm{~s}$ at $95^{\circ} \mathrm{C} ; 40$ cycles of $15 \mathrm{~s}$ at $95^{\circ} \mathrm{C}, 45 \mathrm{~s}$ at $60^{\circ} \mathrm{C}$. Melt curve analyses were conducted to confirm the specificity of amplification products. Relative gene expression was assessed via the $2^{-\triangle \Delta \mathrm{CT}}$ approach [47].

\section{Abbreviations}

YR: Yersinia ruckeri; DEGs: Differentially expressed genes; GO: Gene ontology; KEGG: Kyoto encyclopedia of genes and genomes; ILs: Interleukins; IFNs: Interferons; TNFs: Tumor necrosis factors; CCR9: C-C motif chemokine receptor 9; CARD9: Caspase recruitment domain-containing protein: CASP8: Caspase 8; RIPK1: Receptor-interacting serine/threonine-protein kinase 1-like; IAP: Inhibitor of apoptosis protein-like protein; IKBa: NF-kappa-B inhibitor alpha-like; PPRs: Pattern recognition receptors; HSP90: Heat shock protein 90; NOD1: Nucleotide-binding oligomerization domain-containing protein 1-like; TLRs: Toll-like receptors; TNFAIP3: Tumor necrosis factor alphainduced protein 3-like

\section{Supplementary Information}

The online version contains supplementary material available at https://doi. org/10.1186/s12864-021-07611-4.

\section{Additional file 1. \\ Additional file 2. \\ Additional file 3.}

Additional file 4: Table S1. Characteristics of RNA-seq data.

Additional file 5: Table S2. Summary of information regarding the annotation of new genes.

Additional file 6: Table S3. Information of all the DEGs detected in this study.

Additional file 7: Table S4. The list of immune response-related DEGs identified in this study.

\section{Acknowledgements}

The authors would like to thank Mr. Jian Feng for providing fish materials.

\section{Authors' contributions}

DW and SMS participated in the design of this study, and carried out the experiments. DW drafted the manuscript. SWL provided assistance for data analysis, background information collection and manuscript editing. DFS and TYL acquired the funding, carried out the conception of this study and reviewed the manuscript. All authors have read and approved the content of the manuscript.

\section{Funding}

This study was supported by grants from Central Public-interest Scientific Institution Basal Research Fund, HRFRI (No. HSY202007M) and Central Publicinterest Scientific Institution Basal Research Fund,CAFS (No. 2020TD43). The funding agency did not participate in study design, data collection and analysis, decision to publish, and preparation of the manuscript.

\section{Availability of data and materials}

The dataset(s) supporting the conclusions of this article is(are) included within the article (and its additional file(s). The raw data were available in the NCBI Sequence Read Archive (SRA).

\section{Declarations}

\section{Ethics approval and consent to participate}

The study was carried out in compliance with the ARRIVE guidelines. The Committee of the Ethics on Animal Care and Experiments at Heilongjiang River Fisheries Research Institute of Chinese Academy of Fishery Sciences also approved this study, which was consistent with the Guidelines of European Union Council Directive 2010/63/EU for the protection of research animals.

Consent for publication

Not applicable.

\section{Competing interests}

The authors declare no conflicts of interest.

\section{Author details}

${ }^{1}$ College of Veterinary Medicine, Northeast Agricultural University, 150030 Harbin, China. ${ }^{2}$ Heilongjiang River Fisheries Research Institute, Chinese Academy of Fishery Sciences, 150070 Harbin, China. ${ }^{3}$ Key Laboratory of Aquatic Animal Diseases and Immune Technology of Heilongjiang Province, 150070 Harbin, China.

Received: 7 February 2021 Accepted: 12 April 2021

Published online: 22 April 2021

\section{References}

1. Tobback E, Decostere A, Hermans K, Haesebrouck F, Chiers K. Yersinia ruckeri infections in salmonid fish. J Fish Dis. 2007;30(5):257-68.

2. Tobback E, Decostere A, Hermans K, Ryckaert J, Duchateau L, Haesebrouck F, Chiers K. Route of entry and tissue distribution of Yersinia ruckeri in experimentally infected rainbow trout Oncorhynchus mykiss. Dis Aquat Organ. 2009;84(3):219-28.

3. Jiang J, Zhao W, Xiong Q, Wang K, He Y, Wang J, Chen D, Geng Y, Huang X, Ouyang $P$, Lai W. Immune responses of channel catfish following the stimulation of three recombinant flagellins of Yersinia ruckeri in vitro and in vivo. Dev Comp Immunol. 2017;73:61-71.

4. Li S, Zhang Y, Cao Y, Wang D, Liu H, Lu T. Trancriptome profiles of Amur sturgeon spleen in response to Yersinia ruckeri infection. Fish Shellfish Immunol. 2017;70:451-60.

5. Raida MK, Holten-Andersen L, Buchmann K. Association between Yersinia ruckeri infection, cytokine expression and survival in rainbow trout (Oncorhynchus mykiss). Fish Shellfish Immunol. 2011;30(6):1257-64.

6. Harun NO, Wang T, Secombes CJ. Gene expression profiling in naive and vaccinated rainbow trout after Yersinia ruckeri infection: Insights into the mechanisms of protection seen in vaccinated fish. Vaccine. 2011;29:4388-99.

7. Kumar G, Menanteau-Ledouble S, Saleh M, El-Matbouli M. Yersinia ruckeri, the causative agent of enteric redmouth disease in fish Vet Res. 2015;46(1):103.

8. Zuo S, Karami AM, Ødegård J, Mathiessen H, Marana MH, Jaafar RM, Jørgensen LG, Abdu M, Kania PW, Dalsgaard I, Nielsen T, Buchmann K. Immune gene expression and genome-wide association analysis in rainbow trout with different resistance to Yersinia ruckeri infection. Fish Shellfish Immunol. 2020;106:441-50.

9. Wang Z, Gerstein M, Snyder M. RNA-Seq: a revolutionary tool for transcriptomics. Nat Rev Genet. 2009;10(1):57-63.

10. Long M, Zhao J, Li T, Tafalla C, Zhang Q, Wang X, Gong X, Shen Z, Li A. Transcriptomic and proteomic analyses of splenic immune mechanisms of rainbow trout (Oncorhynchus mykiss) infected by Aeromonas salmonicida subsp. salmonicida. J Proteomics. 2015;122:41-54.

11. Rebl A, Korytar T, Kobis JM, Verleih M, Krasnov A, Jaros J, Kuhn C, Kollner B, Goldammer T. Transcriptome profiling reveals insight into distinct immune responses to Aeromonas salmonicida in gill of two rainbow trout strains. Mar Biotechnol (NY). 2014;16(3):333-48.

12. Purcell MK, Marjara IS, Batts W, Kurath G, Hansen JD. Transcriptome analysis of rainbow trout infected with high and low virulence strains of infectious hematopoietic necrosis virus. Fish Shellfish Immunol. 2011;30(1):84-93.

13. Syahputra K, Kania PW, Al-Jubury A, Jafaar RM, Dirks RP, Buchmanna K. Transcriptomic analysis of immunity in rainbow trout (Oncorhynchus mykiss) gills infected by Ichthyophthirius multifiliis. Fish Shellfish Immunol. 2019;86:486-96. 
14. Wrobel A, Leo JC, Linke D. Overcoming Fish Defences: The Virulence Factors of Yersinia ruckeri. Genes (Basel). 2019;10(9):700.

15. Raida MK, Buchmann K. Development of adaptive immunity in rainbow trout, Oncorhynchus mykiss (Walbaum) surviving an infection with Yersinia ruckeri. Fish Shellfish Immunol. 2008;25(5):533-41.

16. Savan R, Sakai M. Genomics of fish cytokines. Comp Biochem Physiol Part D Genomics Proteomics. 2006;1(1):89-101.

17. Alejo A, Tafalla C. Chemokines in teleost fish species. Dev Comp Immunol. 2011;35:1215-22.

18. Wiens GD, Glenney GW, Lapatra SE, Welch TJ. Identification of novel rainbow trout (Onchorynchus mykiss) chemokines, CXCd1 and CXCd2: mRNA expression after Yersinia ruckeri vaccination and challenge. Immunogenetics. 2006;58(4):308-23.

19. Campuzano A, Castro-Lopez N, Martinez AJ, Olszewski MA, Ganguly A, Wager CL, Hung CY, Wormley Jr FL. CARD9 is required for classical macrophage activation and the induction of protective immunity against pulmonary cryptococcosis. mBio. 2020;11(1):e03005-19.

20. Brown DA, Yang N, Ray SD. Apoptosis in Encyclopedia of Toxicology. 3rd ed. London: Academic; 2014. p.287 - 94

21. Takle H, Andersen $\varnothing$. Caspases and apoptosis in fish. J Fish Biol. 2007;71(sc): 326-49.

22. Reyes-Becerril M, Sanchez V, Delgado K, Guerra K, Velazquez E, Ascencio F, Angulo C. Caspase - 1, -3, -8 and antioxidant enzyme genes are key molecular effectors following Vibrio parahaemolyticus and Aeromonas veronii infection in fish leukocytes. Immunobiology. 2018;223(10):562-76.

23. Karunakaran D, Nguyen MA, Geoffrion M, Vreeken D, Lister Z, Cheng HS, Otte N, Essebier P, Wyatt H, Kandiah JW, Jung R, Alenghat FJ, Mompeon A, Lee R, Pan C, Gordon E, Rasheed A, Lusis AJ, Liu P, Matic LP, Hedin U, Fish JE, Guo L, Kolodgie F, Virmani R, van Gils JM, Rayner KJ. RIPK1 Expression Associates with inflammation in early atherosclerosis in humans and can be therapeutically silenced to reduce NF-kB activation and atherogenesis in mice. Circulation. 2021;143(2):163-77.

24. Hayden MS, Ghosh S. Shared principles in NF-kappaB signaling. Cell. 2008; 132(3):344-62.

25. Sangrador-Vegasa A, Smith TJ, Cairns MY. Cloning and characterization of a homologue of the alpha inhibitor of NF-KB in rainbow trout (Oncorhynchus mykiss). Vet Immuno Immunopathol. 2005;103(1-2):1-7.

26. Zhu L, Nie L, Zhu G, Xiang L, Shao J. Advances in research of fish immunerelevant genes: A comparative overview of innate and adaptive immunity in teleosts. Dev Comp Immunol. 2013;39(1-2):39-62.

27. Jang JH, Kim H, Kim YJ, Cho JH. Molecular cloning and functional analysis of nucleotide-binding oligomerization domain-containing protein 1 in rainbow trout, Oncorhynchus mykiss. Fish Shellfish Immunol. 2016;51:53-63.

28. Paltia Y, Gahr SA, Purcell MK, Hadidi S, Rexroad III CE, Wiensa GD. Identification, characterization and genetic mapping of TLR7, TLR8a1 and TLR8a2 genes in rainbow trout (Oncorhynchus mykiss). Dev Comp Immunol. 2010;34(2):219-33.

29. Baruah K, Ranjan J, Sorgeloos P, MacRae TH, Bossier P. Priming the prophenoloxidase system of Artemia franciscana by heat shock proteins protects against Vibrio campbellii challenge. Fish Shellfish Immunol. 2011;31:134-41.

30. He JY, Wang JR, Xu MS, Wu SW, Liu HH. The cooperative expression of Heat Shock Protein $70 \mathrm{KD}$ and $90 \mathrm{KD}$ gene in juvenile Larimichthys crocea under Vibrio alginolyticus stress. Fish Shellfish Immunol. 2016;58:359-69.

31. Xie Y, Song L, Weng Z, Liu S, Liu Z. Hsp90, Hsp60 and sHsp families of heat shock protein genes in channel catfish and their expression after bacterial infections. Fish Shellfish Immunol. 2015;44(2):642-51.

32. Mérour $E$, Jami $R$, Lamoureux A, Bernard J, Brémont M, Biacchesi S. A20 (tnfaip3) is a negative feedback regulator of RIG-I-Mediated IFN induction in teleost. Fish Shellfish Immunol. 2019:84:857-64.

33. Umasuthan N, Bathige SDNK, Noh JK, Lee J. Gene structure, molecular characterization and transcriptional expression of two p38 isoforms (MAPK11 and MAPK14) from rock bream (Oplegnathus fasciatus). Fish Shellfish Immunol. 2015;47(1):331-43.

34. Krens SG, He S, Spaink HP, Snaar-Jagalska BE. Characterization and expression patterns of the MAPK family in zebrafish. Gene Expr Patterns. 2006; 6:1019-26

35. Guo M, Wei J, Huang X, Zhou Y, Yan Y, Qin Q. JNK1 derived from orangespotted grouper, Epinephelus coioides, involving in the evasion and infection of Singapore grouper iridovirus (SGIV). Front Microbiol. 2016;7: 121.

36. Sadangi S, Mohanty A, Paichha M, Samanta M. Molecular characterization and expression analysis of two crucial MAPKs-jnk1 and erk1 as cellular signal transducers in Labeo rohita in response to PAMPs stimulation and pathogenic invasion. J Fish Biol. 2020;96(3):580-9.

37. Lian HM, Lu TY, Liu HB, Yin JS, Zhang H, Li SW. Isolation, identification and growth of pathogenic bacterium in enteric redmouth disease in rainbow trout Oncorhynchus mykiss. J Dalian Ocean Univ. 2015;30(5):498-504.

38. Li SW, Zhang Y, Cao YS, Wang D, Liu HB, Lu TY. Trancriptome profiles of Amur sturgeon spleen in response to Yersinia ruckeri infection. Fish Shellfish Immunol. 2017;70:451-60.

39. Grabherr MG, Haas BJ, Yassour M, Levin JZ, Thompson DA, Amit I, Adiconis X, Fan L, Raychowdhury R, Zeng Q, Chen Z, Mauceli E, Hacohen N, Gnirke A, Rhind N, Palma F, Birren BW, Nusbaum C, Lindblad-Toh K, Friedman N, Regev A. Full-length transcriptome assembly from RNA-Seq data without a reference genome. Nat Biotechnol. 2011;29:644-52.

40. Tatusov RL, Galperin MY, Natale DA, Koonin EV. The COG database: a tool for genome-scale analysis of protein functions and evolution. Nucleic Acids Res. 2000;28(1):33-6.

41. Kanehisa M, Araki M, Goto S, Hattori M, Hirakawa M, Itoh M, Katayama T, Okuda S, Tokimatsu T, Yamanishi Y. KEGG for linking genomes to life and the environment. Nucleic Acids Res. 2008;36:480-4.

42. Ye J, Fang L, Zheng H, Zhang Y, Chen J, Zhang Z, Wang J, Li S, Li R, Bolund L, Wang J. WEGO: a web tool for plotting GO annotations. Nucleic Acids Res. 2006:34(Web Server issue):W293-7.

43. Li B, Dewey CN. RSEM: accurate transcript quantification from RNA-Seq data with or without a reference genome. BMC. Bioinformatics. 2011;12:323.

44. Kanehisa M, Goto S. KEGG: Kyoto Encyclopedia of Genes and Genomes. Nucleic Acids Res. 2000:28:27-30.

45. Kanehisa M. Toward understanding the origin and evolution of cellular organisms. Protein Sci. 2019;28;1947-51.

46. Kanehisa M, Furumichi M, Sato Y, Ishiguro-Watanabe M, Tanabe M. KEGG: integrating viruses and cellular organisms. Nucleic Acids Res. 2021;49;545 - 51.

47. Livak KJ, Schmittgen TD. Analysis of relative gene expression data using real-time quantitative PCR and the 2(-Delta Delta C(T)) method. Methods. 2001:25(4):402-8.

\section{Publisher's Note}

Springer Nature remains neutral with regard to jurisdictional claims in published maps and institutional affiliations.

Ready to submit your research? Choose BMC and benefit from:

- fast, convenient online submission

- thorough peer review by experienced researchers in your field

- rapid publication on acceptance

- support for research data, including large and complex data types

- gold Open Access which fosters wider collaboration and increased citations

- maximum visibility for your research: over $100 \mathrm{M}$ website views per year

At $\mathrm{BMC}$, research is always in progress.

Learn more biomedcentral.com/submissions 